\title{
PHOTOGRAPHIC RECORDING OF OPACITIES OF THE OCULAR MEDIA*
}

\author{
BY \\ E. F. FINCHAM \\ Institute of Ophthalmology, University of London
}

THE value of photography for recording pathological conditions of the eye is now generally recognized. It is used particularly to show the external parts and such internal surfaces as the iris and fundus. The photography of conditions of the transparent media, like the photography of the fundus, calls for the use of a special optical system, and in the past has not received much attention. Douvas and Allen (1950) described a method of adapting the Nordenson fundus camera for this purpose. The principle of this instrument, based as it is upon the indirect ophthalmoscope, makes it suitable for photographing the media by transillumination. Diseases affecting the media of the eye usually result in some loss of transparency, causing reduction in vision. The possibility that these conditions may be progressive, so often the case when the crystalline lens is affected, makes the keeping of accurate records of great importance. The much debated questions of the possibility of therapeutic treatment of some forms of cataract, or the retardation of its development by the control of diabetes, the supply of a deficient hormone in endocrine failure, or the removal of focal infections, call for the comparison of records made over a period of time.

In recording the progress of cataract, reliance cannot be placed upon the measurement of the patients' vision. The early stages of the disease may be detected by means of the ophthalmoscope or slit lamp while vision is still normal, and when vision does become affected its reduction bears no definite relation to the extent of the opacity. Any graphic records, to be useful for comparison from time to time, must give an accurate representation of fine detail; drawings cannot be relied upon in this respect, and, moreover, photography is much more rapid. It is well known that the different appearances which opacities of the anterior media assume with slightly differing methods of illumination are of great help in diagnosis. Unfortunately, in static photography the use of these variations in lighting is seldom possible

* Received for publication October 29, 1954. 
and it is necessary to adopt a simple system of lighting and observation that can always be repeated. For our purpose the method of transillumination by light reflected from the fundus is the most satisfactory. It has the advantage that the whole pupillary space can be shown in one photograph with approximately uniform lighting. Other methods, such as slit-lamp illumination, would require a number of photographs with adjustment of the illumination to explore the field and no certainty that the conditions could be repeated.

\section{Method}

The principle of examination of the media by transillumination with the ophthalmoscope is well known. The illuminating system with its axis coincident with the line of observation remains the same as for fundus examination, and the lens required to focus the plane of the medium to be viewed is brought into the sight-hole. In photography a much larger aperture than the ophthalmoscope sight-hole is required; a transparent mirror through which the photograph can be taken, therefore, replaces the usual perforated mirror. This entails considerable loss of light, so that an otherwise efficient illuminating system must be used; the slit lamp is almost ideal for this purpose. The general principle of slit-lamp examination does not call for provision in the apparatus for alignment of the illuminating and observing axes; this, however, is desirable with the use of the Hruby lens, and with some modern slit lamps such an alignment can be made. For the present purpose the A.I.M. slit lamp has been found very convenient.

In this instrument the projection system is vertical, the light being reflected along a horizontal axis by a mirror (or prism) immediately following the projection lens. This mirror is mounted on a cap which can be lifted from the lens-mount and replaced by a similar cap bearing a transparent mirror. This is simply a plate of glass about $1 \mathrm{~mm}$. thick and of sufficient dimensions to allow a clear view for the camera, i.e. about $5 \mathrm{~cm}$. high by $4 \mathrm{~cm}$. wide. A small reflex camera replaces the microscope. The camera is of fixed focus for an object distance of $10 \mathrm{~cm}$. and gives a magnification in the image of 1.5 diameters. A lens of $2 \frac{1}{4}$ " focal length is used. The construction of the camera consists of a reflex viewing system attached to the body of a Contax camera containing a focal-plane shutter. The shutter and mirror are operated by two synchronized cable releases. The reflex image is viewed in the horizontal direction by means of a reflecting prism and eye-piece. Focusing and centring are achieved by moving the whole stand carrying slit lamp and camera by the joy-stick control provided on this apparatus. The slit is used at its maximum width and provision has been made to over-run the lamp by 2 volts above its normal 6-volt rating, though this extra light is not required when the media are reasonably clear and the pupil is well dilated. Suitable photographic materials are Ilford H.P. 3 and Kodak Super XX; both $35 \mathrm{~mm}$. films and plates have been used. The latter, used with the Contax plate back, are preferred because usually only a few exposures are required at a time. Exposures of $0.1 \mathrm{sec}$. at a marked aperture of F4 are satisfactory. The general arrangement of the apparatus is shown in Fig. 1 (opposite).

The lesions which it is required to record may extend over some depth within the media, such as the crystalline lens, and thus the question of depth of field in the photograph must be considered. Owing to the magnification that is required, the depth of field is small, and, moreover, the light available does not allow the use of a small aperture in the camera lens. Hence we cannot produce results with the whole thickness of the crystalline lens in focus. Even if such a depth of field could be obtained the photographs would give no indication of the relative depths of lesions and therefore in most cases it is preferable to work with a large aperture 


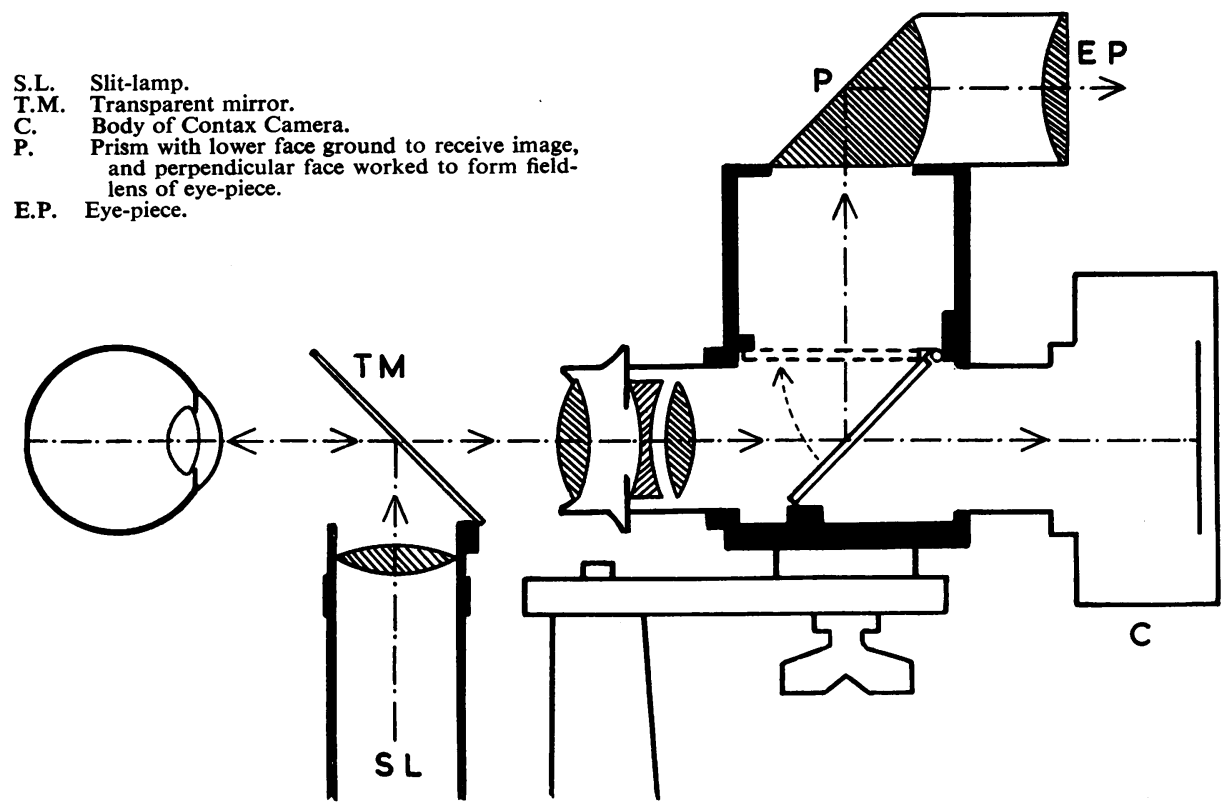

FIG. 1.-Diagram of apparatus.

lens and to focus upon a selected part. This procedure was followed in the examples shown in Fig. 2 (overleaf). If necessary two or more exposures with different adjustments of focus may be made.

Fig. 3 (overleaf) shows records of a case in which a fragment of steel passed through the crystalline lens. By taking two photographs, one focused on the anterior capsule and one on the posterior, the damage in both regions is recorded and differentiated.

A difficulty which occurs with the examination of the media by transillumination is that the illumination of the fundus against which opacities of the media are to be viewed is hindered by the presence of those opacities. Thus the efficiency of the method decreases as the opacities become denser and more extensive. In addition to this, the lesions are opaque because they scatter light; parts which are dark by transillumination are light by focal illumination. Therefore, unless we can avoid the light striking these opacities as it enters the eye, the contrast of their darkness against the luminous background of the fundus will be reduced or lost altogether. The use of the localized beam of the slit lamp is some help in this respect. It is sometimes possible to manipulate the apparatus so that direct illumination of the opacity is avoided. A distinct advantage is also gained by the use of a deep orange filter (Ilford Micro 5) in either the illuminating or photographic system. The orange light from the fundus passes the filter while much of the white light scattered by the media and particularly by the opacities is absorbed, and thus the contrast is increased. The effect of direct illumination of a dense opacity by the light entering the eye is shown in Fig. 2(c). In this case the opacity occupied such a large part of the pupil that it was not possible to illuminate the fundus without some light striking the opacity; hence it is seen with the lower part illuminated directly and the upper silhouetted against the fundus. 


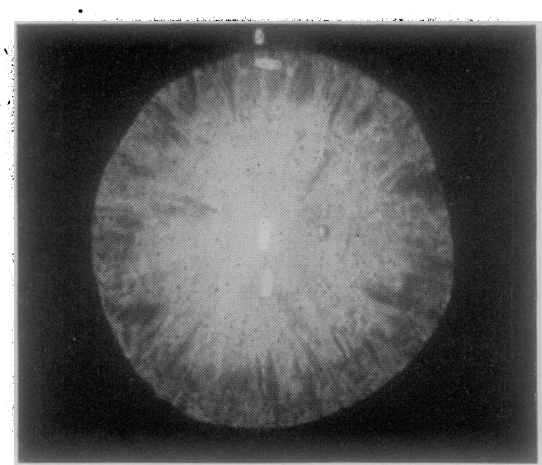

(a)

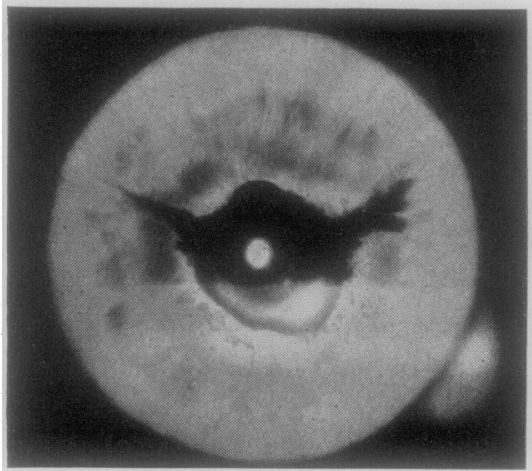

(c)

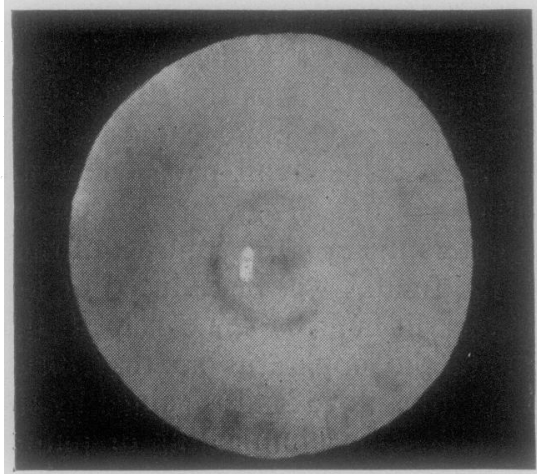

(e)

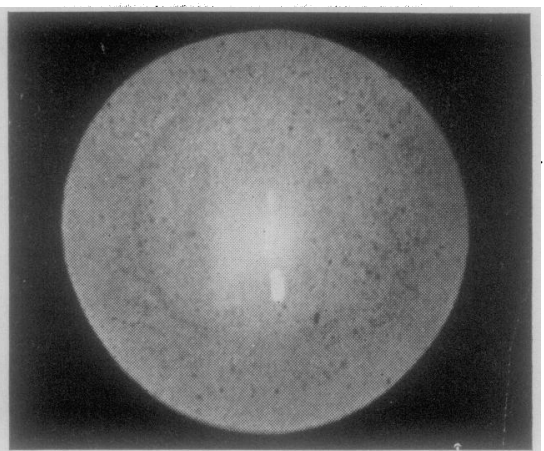

(b)

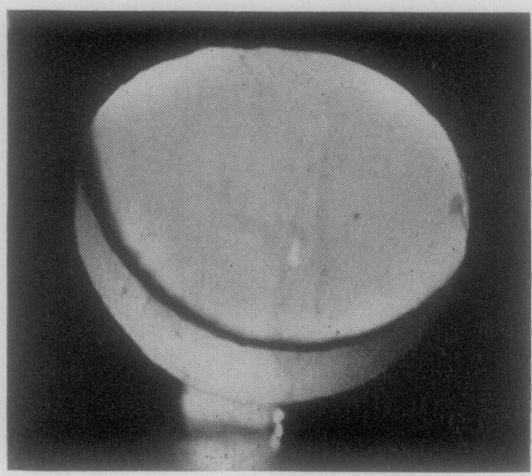

(d)

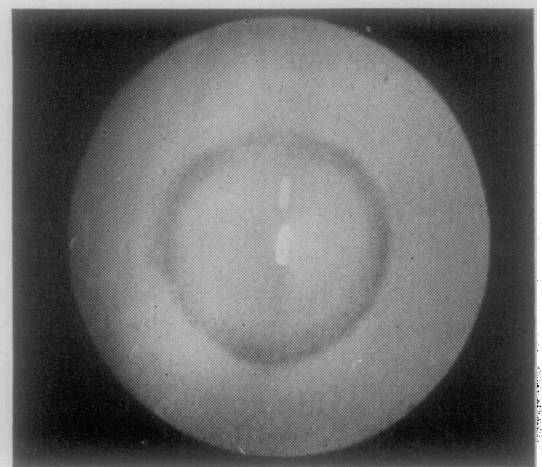

$(f)$

Fig. 2.-Six examples of photographic recordings.

(a) Female, aged 70, senile cataract.

(b) Female, aged 16, minute punctate opacities.

(c) Male, aged 31, extensive posterior polar, and lamellar opacity.

(d) Female, aged 10, subluxation of lens.

(e) Female, aged 39, blue spot and nuclear cataract.

$(f)$ Female, aged 11 (daughter of $(e)$ ), congenital zonular cataract. 


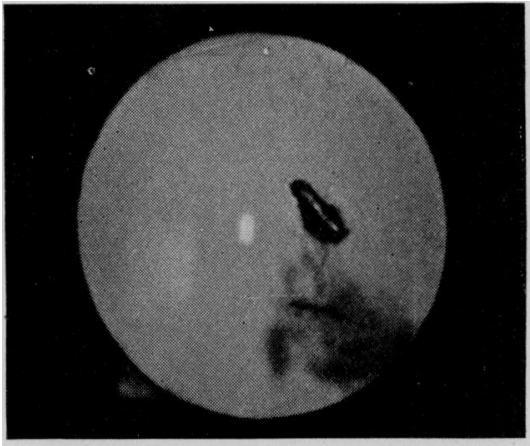

(a) $\mathrm{A}$

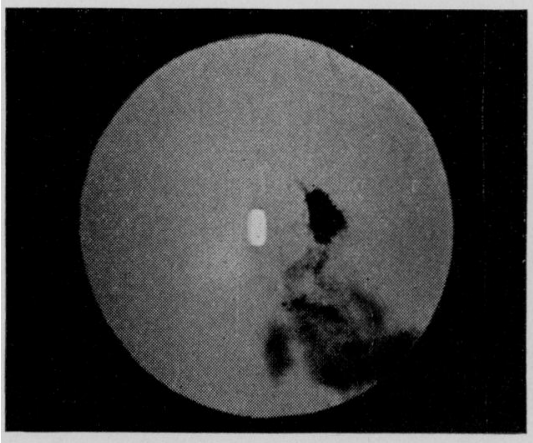

(b) $\mathrm{A}$

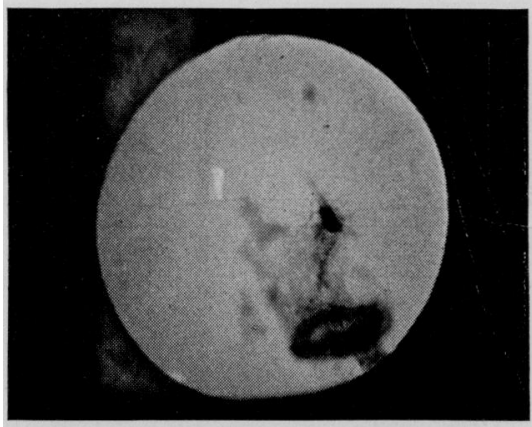

(c) A

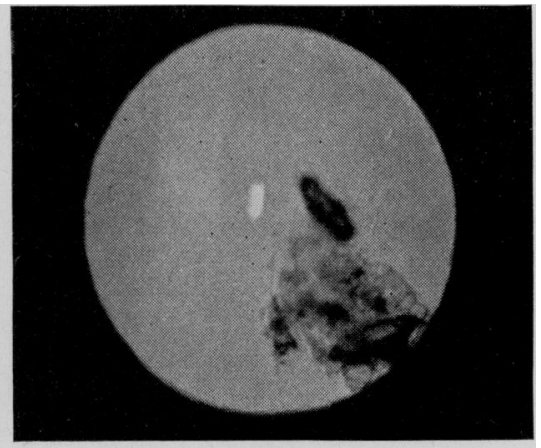

(a) $\mathrm{P}$

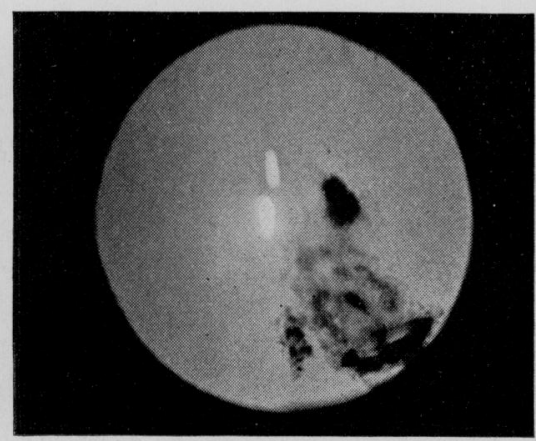

(b) $\mathrm{P}$

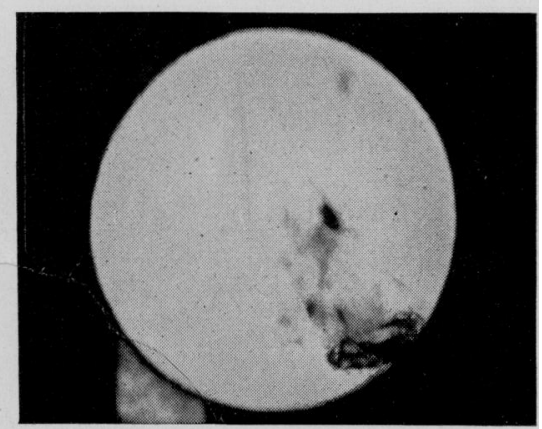

(c) $\mathbf{P}$

Fig. 3.-Progress of a trauma in a male patient aged 26; a fragment of steel passed through the lens and was removed from the vitreous.

(A) Camera focused on anterior capsule. (P) Camera focused on posterior capsule.

(a) 19 days after accident.

(b) 35 days after accident.

(c) 117 days after accident

I wish to thank Mr. N. Roberts and Mr. F. Sheen for their skilled work in adapting the camera to the slit lamp; thanks are also due to Miss M. Pugh, and to Messrs E. M. Ayoub, J. H. Dobree, D. P. Greaves and J. H. Redmond Smith for referring the cases which were photographed.

\section{REFERENCE}

Douvas, N., and Allen, L. (1950). Amer. J. Ophthal., 33, 291. 